\title{
SIG NA GESTÃo DA ÁGUA PARA A AGRICULTURA
}

\author{
António Perdigão ${ }^{1}$
}

\begin{abstract}
Resumo - As necessidades decorrentes da aplicação da Reforma da PAC e da Directiva Quadro da Água da UE exigem um conhecimento, tão preciso quanto possível, da utilização da água, em termos de quantidade, disponibilidade e qualidade, de forma a possibilitar uma gestão sustentada e eco-compatível dos recursos hídricos, nos países do Sul da Europa. Essas necessidades têm vindo gradualmente a exigir o recurso a meios, que envolvem Tecnologias da Informação, Sistemas de Monitorização e acompanhamento, utilizando SIG, algoritmos, modelos de simulação e Detecção Remota, com vários níveis de resolução espaço-temporal. As características técnicas dos utensílios utilizados muitas vezes não se encontram adaptadas ao contexto das unidades de paisagem e das entidades administrativoregionais a que serão aplicadas. A identificação e caracterização das designadas unidades de paisagem envolve uma avaliação do conteúdo temático dum determinado espaço, baseado na sua utilização e ocupação do solo, organizada como unidade espacial.
\end{abstract}

Palavras-chave: Detecção Remota, gestão da água, tecnologias da informação, agricultura, regadios, fenologia.

Abstract - GIS in water management for agricultural pURposes. The needs originating from the application of European Union's CAP and Water Directive demand a very precise knowledge of the potential uses of water, especially in what concerns to its volumes, availability and quality. In Southern Europe this is particularly significant in order to conduct an eco-compatible management of water resources. Decision Support Systems (DSS) for agricultural purposes and management of agriculture vs. environment conflicts, assuming the need for a compatible water management, account for the objectives and tools adapted to the problems under analysis. The specificities of agricultural practices have been slowly requiring the use of information technology tools, monitoring and follow-up systems, GIS, simulation models and remote sensing approaches, using platforms with different spatial and temporal resolutions. Irrigation management involve availability of good quality data assuming that most information is dynamic, therefore requiring permanent updating (e.g. land use/land cover and climate data). However, the technical characteristics of the tools, very seldom are not adapted to

1 Engenheiro Agrónomo. Chefe da Divisão de Cartografia e Informação Geográfica do IDRHa-MADRP, Av. Afonso Costa n. ${ }^{\circ}$ 3, 1949-002 Lisboa. Tel: +351218 442 381. Fax: 351218442411. 
the landscape units context or to the administrative/regional units, to which they are to be applied. The land units approach enables the thematic analysis of landscapes, assuming a strategy based on textures classified as spatial units, in terms of land use/land cover characteristics.

Key words: Remote sensing, water management, information technology, agriculture, irrigation, phenology.

Resumé - Systèmes d'INFORMATION GÉOGRAPHIQUe DESTINÉS À LA GESTION DE L'EAU pour l'Agriculture. L'application de la Réforme de la Politique Agricole Commune et de la Directive Cadre Européenne de l'Eau exige une connaissance précise de l'usage de l'eau pour l'agriculture dans les pays du Sud de l'Europe (sa quantité, disponibilité et qualité), afin de permettre une gestion durable des ressources hydriques. Ce qui implique l'utilisation croissante des méthodologies de type SIG, appliquées à différents niveaux de résolution, spatiaux et temporels. Ces méthodologies doivent être adaptées au contexte des unités de paysage et des divisions administratives concernées.

Mots-clés: Télédétection, gestion de l'eau, techniques d'information, agriculture, irrigation, phénologie.

\section{INTRODUÇÃO}

Os países do Sul da Europa são particularmente afectados pelos problemas relacionados com uma utilização não controlada dos recursos hídricos, provocando conflitos entre os diferentes utilizadores.

A implementação dos processos de apoio à decisão no sector agrícola tendo em conta as necessidades decorrentes duma gestão sustentada e eco-compatível dos recursos hídricos envolve: a definição clara dos objectivos; a escolha de instrumentos adaptados à resolução espacial adequada às necessidades, tendo em conta os diferentes níveis de abordagem dos problemas; e a adequada monitorização para uma avaliação do seu impacto e da potencialidade do utensílio seleccionado.

As necessidades específicas da agricultura têm vindo a exigir gradualmente, a utilização de meios que envolvem Tecnologias da Informação, sistemas de monitorização e acompanhamento, SIG (Sistemas de Informação Geográfica) e Detecção Remota com vários níveis de resolução espaço-temporal.

A Detecção Remota deve ser entendida como uma tecnologia que possibilita a obtenção de dados sobre diferentes aspectos relacionados com a ocupação do solo, tendo como base o conhecimento dos calendários fenológicos e da campanha de rega das culturas mais relevantes da área em estudo, na base duma escolha criteriosa das respectivas coberturas.

A gestão da rega necessita de informação temática a obter de várias fontes, uma parte da qual tem um carácter não dinâmico, necessitando de actualização frequentes (dinâmica da utilização dos solos, clima, cadastro, tipo e sistema de rega). 
A identificação e caracterização das designadas unidades de paisagem, envolve a análise discriminatória do conteúdo temático dum determinado espaço, com uma dominante texturada, caracterizando os diversos territórios em termos da sua ocupação do solo, organizada como nível de unidade espacial.

\section{A PROBLEMÁTICA DA UTILIZAÇÃO DA ÁGUA PARA A AGRICULTURA E A UTILIZAÇÃO DE PLATAFORMAS DE OBSERVAÇÃO DA TERRA PARA UMA MELHOR GESTÃO DO TERRITÓRIO}

\section{A realidade dos aproveitamentos hidroagrícolas}

As Associações de Beneficiários (AB), com uma importância acrescida na gestão da água nas áreas abrangidas pelos Aproveitamentos Hidroagrícolas ( $\mathrm{AH}$ ), desempenham cada vez mais um papel chave na gestão desse mesmo recurso.

A utilização de SIG, para um adequado armazenamento e análise dos diferentes dados relacionados com a agricultura (bases de dados e cartografia

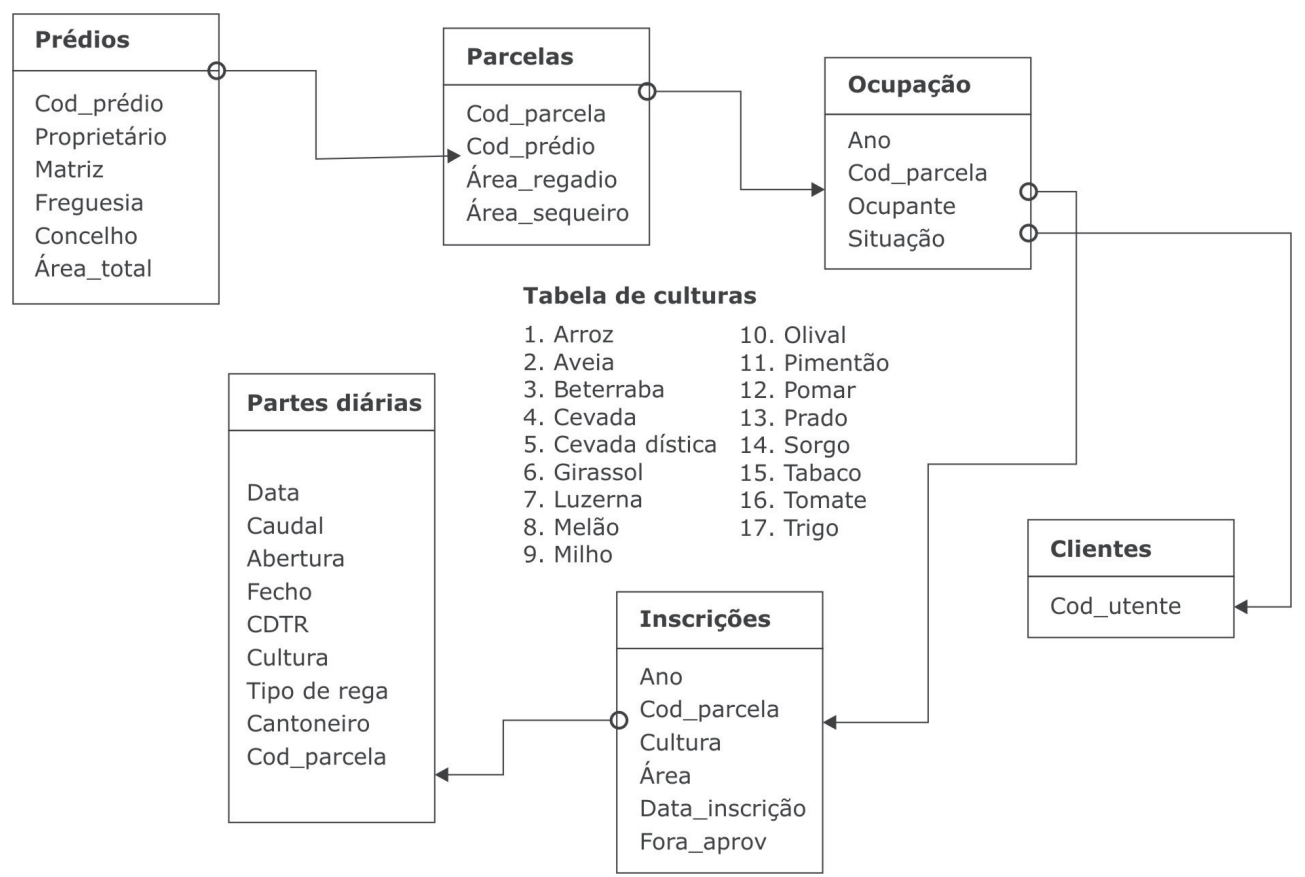

Fig. 1 - Exemplo da estrutura da Base de Dados de Gestão do sIGHAC (Sistema de Informação Geográfica do Aproveitamento Hidroagrícola do Caia) (Perdigão et al., 2001).

Fig. 1 - Example of the structure of the SIGHAC Database (Geographic Information System for the hydro-agricultural management of the Caia) (PERDIGÃO et al., 2001). 
temática relacionada com os regantes), exemplo de estrutura que se apresenta na figura 3, bem como o recurso a sistemas de processamento de imagens integrados com SIG, permite: fornecer informações aos regantes sobre quando e que quantidades de água devem aplicar a cada cultura, tendo em conta o tipo de solo; comparar os volumes de água fornecidos com os previstos, de forma a adaptá-los ao longo da campanha de rega; conhecer a distribuição espacial das actividades agrícolas ao longo do ano e da evolução das culturas ao longo da campanha de rega; apoiar a estimativa antecipada da produção, tendo em conta as características hidrológicas do ano e o seu impacto nas culturas.

A complexidade e a peculiaridade das diferentes situações implica a constituição e implementação duma Base de Dados Digital dos Regantes específica de cada Aproveitamento Hidroagrícola, exemplo que se apresenta na figura $1 \mathrm{em}$ relação à ABCaia, contendo informação sobre as actividades agrícolas desses mesmos regantes de forma a permitir: a identificação e o armazenamento de alterações dos limites das parcelas agrícolas e do seu conteúdo temático; a elaboração de documentação para utilização administrativa; o fornecimento de estatísticas a diferentes níveis administrativos e sobre diferentes temáticas; e o apoio à gestão tendo em vista um melhor acompanhamento da campanha de rega.

\section{Constituição e estruturação da informação cartográfica de apoio}

A paisagem agrícola tem como base o tipo e a estrutura da exploração agrícola em que está inserida, num conjunto de paisagens com continuidade e com a mesma utilização. Na vertente específica da utilização da água para a agricultura podem considerar-se dois níveis de abordagem para a paisagem agrícola, organizados em: Associações de Beneficiários, como entidades territoriais de gestão dos recursos hídricos, ou em Explorações Agrícolas, como estruturas que exercem uma acção sobre o território.

A discriminação das unidades de paisagem a partir de imagens de satélite de alta resolução tem em conta a tipologia dos seguintes elementos: ocupação/ /utilização do solo; organização espacial desses mesmos dados, com base na sua estrutura parcelar, densidade e repartição espacial.

A análise discriminatória do conteúdo temático da imagem de satélite efectua-se na base de vários níveis de segmentação: um primeiro, baseado na análise dum espaço com uma dominante estruturada, caracterizando o território; um segundo, que define a ocupação do solo, em termos da sua organização; um terceiro, que envolve a designada unidade de paisagem, entendida como unidade espacial.

A uma determinada paisagem corresponde, por outro lado, um certo número de elementos que podem ser identificados a partir de plataformas espaciais, como é o caso: das parcelas agrícolas, caracterizadas por descritores (forma, dimensão, orientação, limites); das redes constituídas por linhas mais ou menos estruturantes da paisagem, canais de rega, rede de enxugo, rede de estradas e caminhos; das áreas construídas, agrupadas ou dispersas. 
A problemática da gestão da rega pode ser estruturada nas seguintes categorias, onde cada uma desenvolve acções específicas, de acordo com os temas apresentados cuja dinâmica pode ser verificada na figura 2: objectivo (definição das políticas de rega); condições (estabelecimento das condições, instalação de sistemas, aspectos institucionais); processos (controlo dos processos, gestão da água, culturas, aspectos institucionais e facilidades financeiras); contexto (desenvolvimento de actividades compatíveis, restrições económicas e sociais específicas); melhorias (melhoria do sistema e das facilidades físicas).

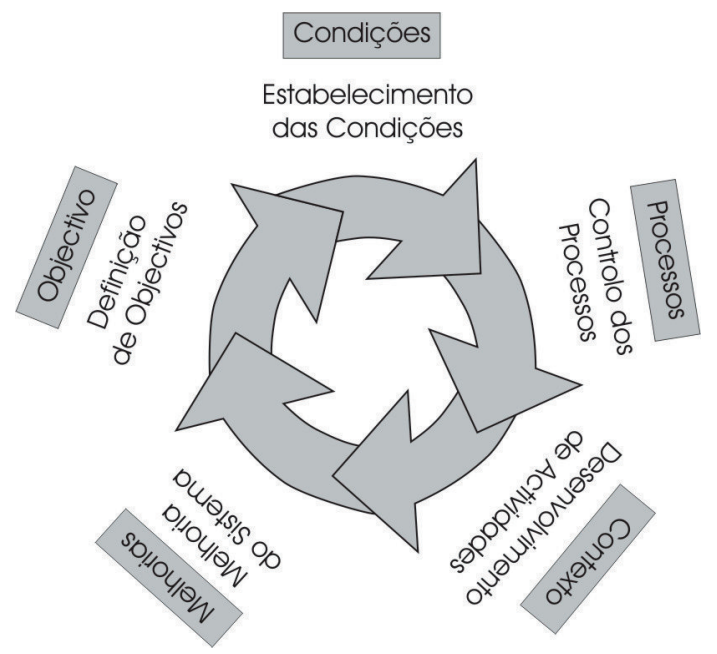

Fig. 2 - Diagrama da problemática da gestão de rega.

Fig. 2 - Schematic presentation of the problems involved in irrigation management.

\section{Tecnologias SIG e modelos de desenvolvimento e apoio}

A Detecção Remota deve ser considerada como uma tecnologia utilizada para obtenção de dados sobre ocupação e utilização do solo, relevante para as seguintes componentes de gestão: água (necessidades hídricas das culturas, áreas com problemas específicos ligados aos solos); cultura (área de intervenção, sua distribuição geográfica, uniformidade ou não do seu desenvolvimento); aspectos institucionais (área efectivamente regada versus área com direitos atribuídos, do qual se apresenta exemplo na figura 4); aspectos financeiros (taxas de rega, função dos consumos ou área coberta pela infra-estrutura).

De tudo o atrás referenciado sobressai a importância de efectuar a escolha das imagens em função dos calendários fenológicos e de rega das culturas mais importantes presentes na área de estudo, tendo em conta: a dimensão das parcelas; os calendários fenológicos das diferentes culturas, na sua vertente relação fisiologia vegetal, dinâmica solo/água e características espectrais das mesmas; 


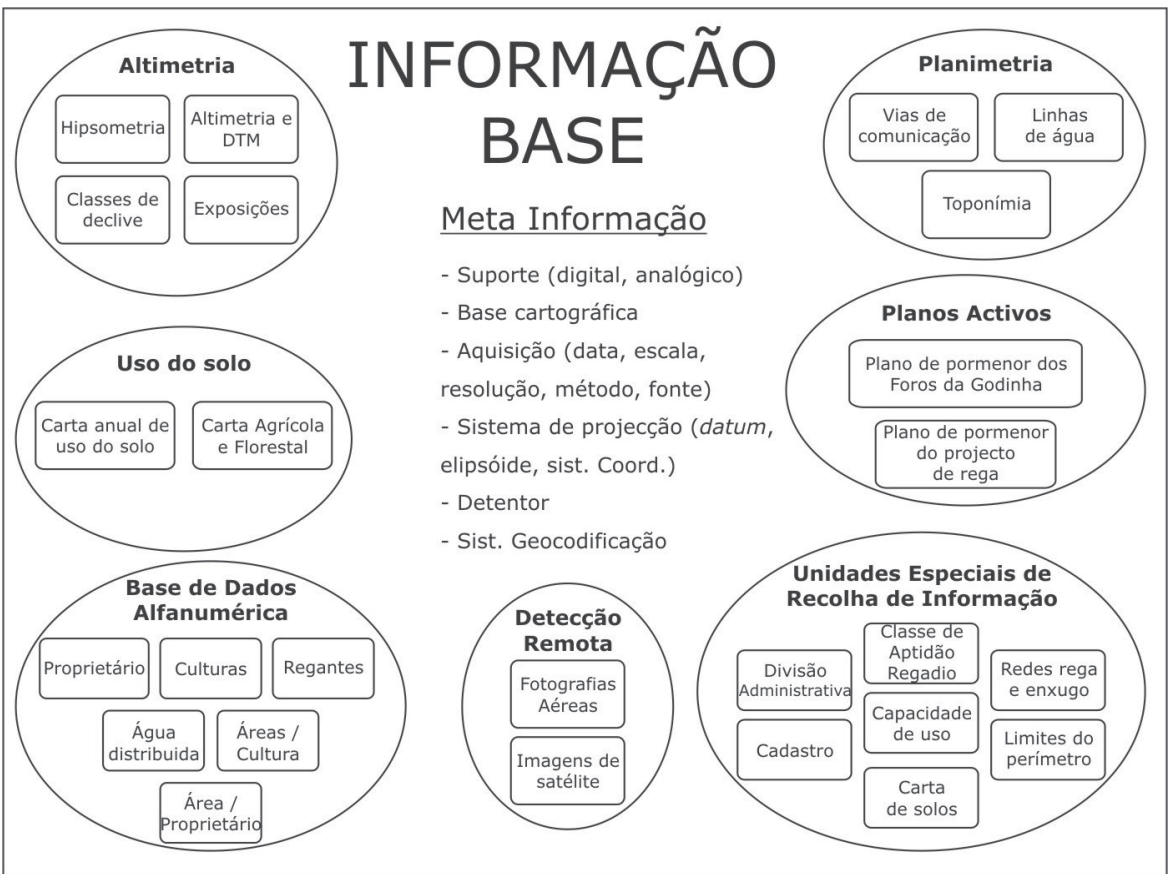

Fig. 3 - Organigrama estrutural de informação de base do SIGHAC

(Fonte: Perdigão et al., 2001).

Fig. 3 - Structural diagram of the basic data required by the SIGHAC (Source: PERDIGÃO et al., 2001).

e a possibilidade de nos virmos a confrontar com potenciais situações de confusão espectral entre as diferentes culturas regadas e destas com culturas de sequeiro.

Importa ainda referir que a qualidade dum trabalho de inventário temático, sobre a utilização do solo, depende de vários factores, nos quais se incluem a metodologia de trabalho, o conhecimento dos calendários culturais e das características fenológicas das culturas, a disponibilidade de informação temática complementar e do trabalho de apoio de campo, bem como do conhecimento efectivo do desenvolvimento dessas mesmas culturas para o período em análise, tendo em conta o tipo de ano hidrológico e a região agro-ecológica em que se insere.

A segmentação de qualquer unidade de paisagem envolve uma diferenciação e estruturação da paisagem em unidades com igual aparência, na base de muitos elementos subjectivos, os quais necessitam de ser justificados ou modificados pela análise do conteúdo dessas mesmas unidades. Cada objecto deve, pois, apresentar uma relação dinâmica com os diferentes objectos presentes na mesma unidade geográfica, cuja presença no espaço e no tempo possibilitará a identificação das diferentes manchas e texturas presentes na imagem de satélite, as quais são identificadas a partir de descritores visuais, que permitem a sua caracterização, agrupamento e diferenciação. 
O diagnóstico da imagem depende dum conjunto de entidades espaciais, identificadas de acordo com o seu conteúdo, os seus descritores morfológicos, a sua cor, a densidade do seu descritor espectral e com a combinação dos diferentes elementos texturais presentes.

Na metodologia de identificação de unidades de paisagem, dado não existir nenhum nível espaço-temporal universal e individualizado a priori, deve intervir, obrigatoriamente, o conhecimento de elementos auxiliares não observáveis na imagem, como, por exemplo, o conhecimento das características do terreno, da sua morfologia e de outros elementos relacionados.

Esta operação designada por zonagem, deve ser entendida como uma divisão espacial em conjuntos definidos com base numa organização interna de caracteres físicos e antrópicos, com níveis integrados.

Esta zonagem deve ser estruturada tendo em conta os dois níveis envolventes: a pequena região, definida em termos dos sistemas de ocupação e utilização do solo, envolvendo conjuntos de território que apresentam uma certa unidade; a terra que abrange espaços agrícolas, urbanos, peri-urbanos ou naturais, correspondendo a um 'território' relativamente homogéneo, delimitando um determinado espaço, reflexo duma determinada organização da estrutura agrária.

A análise da ocupação ou utilização principal do solo da unidade designada por terra permite a criação da tipologia espacial e temática dos diferentes sistemas de organização do espaço, a qual poderá ser definida com base numa grelha de caracteres, tendo em conta: o índice de artificialização; o índice de abandono; o índice de pressões internas ou externas.

Este tipo de análise permite a avaliação da importância dos trabalhos a realizar, tendo em conta a diversidade do espaço em análise, na base: dos contrastes topográficos que vão influenciar os procedimentos de pré-processamento da imagem; das variedades das organizações espaciais que aumentam a complexidade dos procedimentos de processamento e das deduções interpretativas da imagem.

A utilização de dados obtidos por Detecção Remota permite um melhor conhecimento específico do território, através de: uma abordagem global, tendo em conta a continuidade espacial, dado poder ser observada uma unidade geográfica contínua; uma informação exaustiva sobre o conteúdo temático de ocupação do solo, tendo em conta a resolução espacial das imagens escolhidas e da escala de trabalho; uma melhor informação sobre os objectos a identificar, através da utilização de bandas espectrais alargadas; a obtenção de informação temática sobre as mudanças intra-anuais dos fenómenos (meteo-vegetação-erosão) e das dinâmicas inter-anuais dos diversos sistemas espaciais.

A análise duma imagem de satélite envolve uma cadeia de raciocínios, ou seja a formalização a partir dum diagnóstico do ambiente que nos envolve, do sistema espacial que o integra e do seu funcionamento; construção lógica, que organiza e estrutura, na forma de modelo conceptual, os dados necessários à cadeia de raciocínio; construção física que não é mais que a transcrição estruturada das diversas informações. 


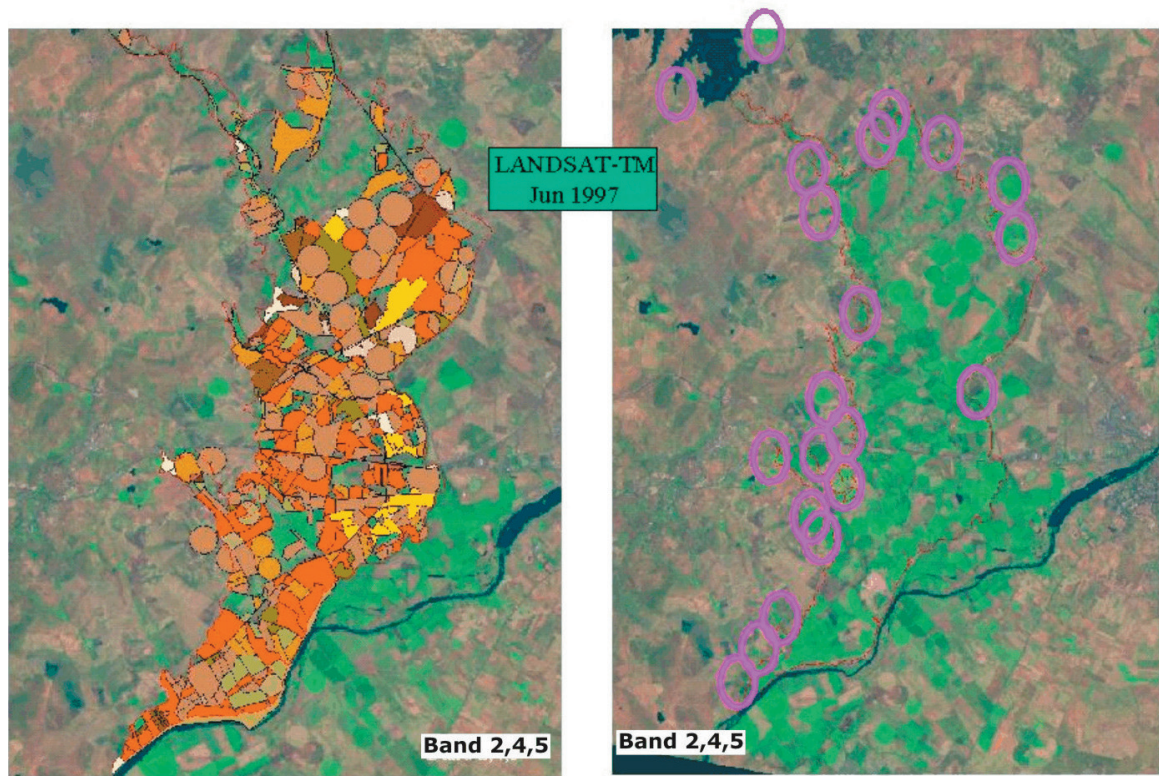

Fig. 4 - Utilização de imagens Landsat TM para a) o Inventário cultural e b) Gestão e identificação de regantes a título precário, no Aproveitamento Hidroagrícola do Caia (Oliveira, 2000).

Fig. 4 - Use of Landsat TM Images for Crop Survey (a) and Management and Detection of Irrigation Systems (b) in the Hydroagricultural Management of the Caia (OLIVEIRA, 2001).

\section{A utilização de dados de satélite de baixa e alta resolução na identi- ficação de parâmetros agro-meteorológicos relacionados com a gestão de água}

A utilização de satélites multiespectrais permite o desenvolvimento de métodos clássicos de determinação de importantes parâmetros agro-meteorológicos, directamente relacionados com um aumento da resolução espacial de metros para quilómetros, bem como da sua resolução temporal, a qual pode variar de horas a um período mais vasto.

No entanto, a nova geração de sensores espaciais da EUMETSAT (Organização Europeia para a Exploração de Satélites Meteorológicos) representa uma grande evolução, dado permitirem um melhor conhecimento dos processos relacionados com a superfície terrestre, nos quais se enquadram o SEVIRI, instalado no MSG, O AVHRR-3 a bordo dos NOAA e o futuro EPS/METOP, os quais possibilitarão um acompanhamento diário do desenvolvimento vegetativo das culturas.

Um dos problemas da utilização de tecnologias de Observação da Terra está relacionado com a falta de adequação da resolução espacio/temporal das 
imagens actualmente disponíveis (ASTER, IRS-C, IRS-D, LANDSAT 5 E 7, SPOT, IKONOS E QUICKBIRD) face às necessidades dos utilizadores dos satélites de alta resolução, nomeadamente na interligação entre os calendários de passagem do satélite e o das culturas a inventariar e, particularmente, no caso vertente, as de regadio e especificamente os períodos de grande desenvolvimento vegetativo.

A potencial utilização de novas plataformas de Observação da Terra, nomeadamente a ASTER (Advanced Spaceborne Thermal Emission and Reflection Radiometer) instalada no satélite TERRA, em Dezembro de 1999 pela NASA, com uma resolução espacial de $15 \mathrm{~m}$ no vNIR (Very Near Infra-Red), de 30m no swIR (Short Wave Infra-Red) e de $90 \mathrm{~m}$ no Infra-Vermelho Térmico, possibilitará a obtenção de mapas de Kcs (Coeficientes Culturais), cujo exemplo pode ser observado na figura 5, com uma melhor resolução espacial, bem como a possibilidade de investigação de métodos para obtenção de novos dados radiométricos, nomeadamente na gama do Infra-Vermelho de onda curta e no Térmico.

Actualmente existem várias abordagens para a obtenção de cartas dos parâmetros de Kc, a partir de Sistemas de Observação da Terra (D’URso, 2001; Menenti, 2000) que possibilitam o conhecimento das necessidades hídricas das culturas, nomeadamente usando como base dados de NDVI, na gama dos comprimentos de onda do Vermelho e Infra-Vermelho próximo.
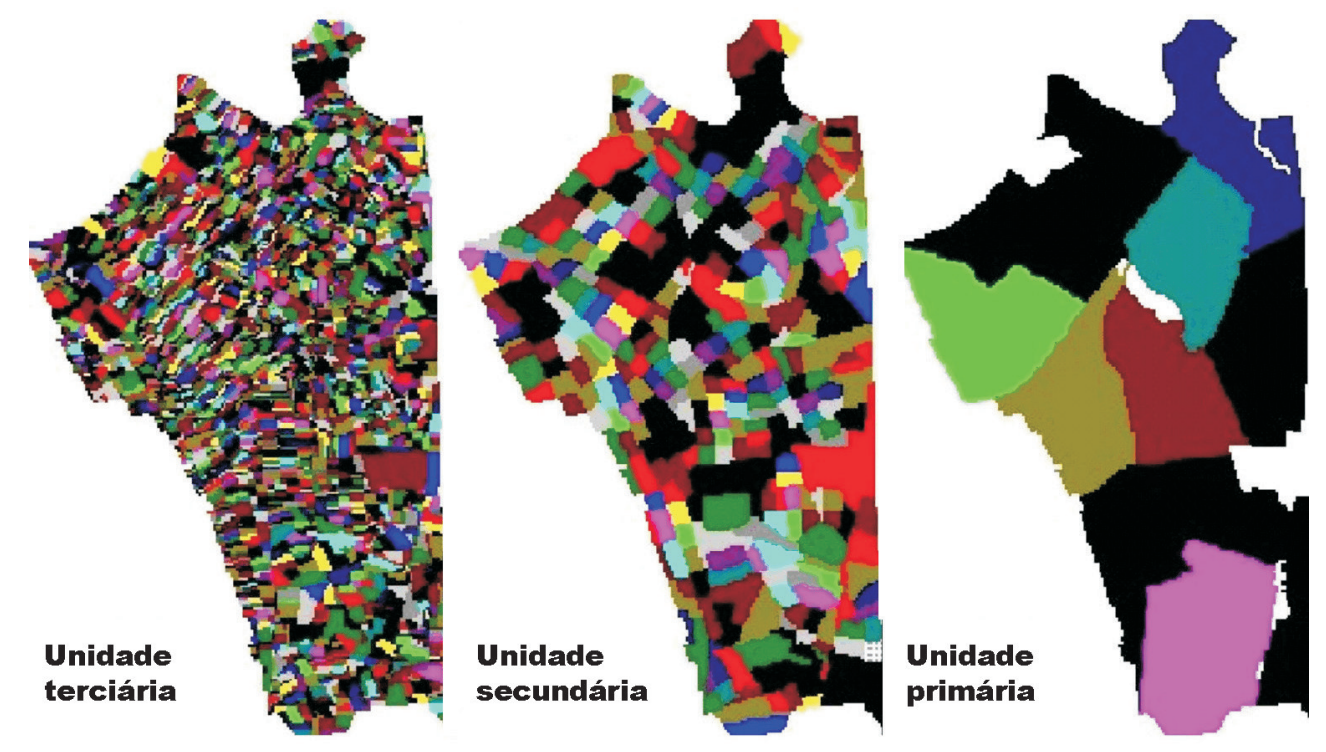

Fig. 5 - Aplicações do modelo sebal ao Inventário da Eficiência de Rega, para várias unidades de abordagem - área de Paestum - Itália

(Montesinos \& Castaño, 1999).

Fig. 5 - Use of the SEBAL Model for Surveying Irrigation Efficiency at Various Levels of Analysis; Paestum Area, Italy (Source ASTIMWR, 1999). 
Uma outra abordagem envolve a utilização de modelos de transporte de água no sistema solo-planta-atmosfera, usando o conceito de coeficiente cultural, como relação entre a evapotranspiração da cultura e o valor da evapotranspiração de referência (ALLEN et al., 1998), em que as duas quantidades são expressas em termos da equação de Penman-Monteith (Menenti, 2000), considerando o Kc, como uma função analítica dum conjunto de parâmetros como por exemplo o Índice de Absorção Foliar.

O SEBAL - Surface Energy Balance Algorithm for Land Surfaces - (BASTIAANSSEN e VISSER, 1996), determina valores de ETR (Evapotranspiração Real), através duma equação de Balanço de Energia, pixel a pixel, com base em teorias micro-meteorológicas sem ter em conta a classificação da cultura, recorrendo a imagens de baixa resolução.

A temperatura de superfície é interpretada nos estudos de energia como resultado da posição da energia bruta entre o fluxo calórico latente e o sensível, sendo no algoritmo SEBAL a diferença entre a temperatura de superfície e a do ar.

Os mapas de evapotranspiração, obtidos a partir de satélites de alta-resolução, fornecem informação espacial detalhada sobre o estado hídrico das culturas, valores esses que variam tendo em conta as condições de cultivo (regada ou não) e as condições físicas da mesma, como se pode ver no exemplo apresentado na figura 5 .

O estado de desenvolvimento vegetativo e a cobertura do solo têm um papel importante na escala global de processos, assumindo-se que a vegetação influencia fortemente o clima em termos do balanço de energia e, ao mesmo tempo, representa um indicador sensível dos efeitos das mudanças climáticas e das pressões antropogénicas, tendo as técnicas de Detecção Remota sido desenvolvidas e testadas para a obtenção de indicadores relacionados com os parâmetros físicos da vegetação.

\section{CONSIDERAÇÕES FINAIS}

A complexidade da temática abordada, bem como as diferentes implicações legais e jurídicas da utilização da informação derivada, faz ressaltar a importância dum conhecimento preciso da informação cartográfica a utilizar, da sua fonte, do seu conteúdo, dos metadados associados, sua exactidão e precisão, bem como sobre a complexidade dos conceitos, nomeadamente no seu enquadramento no tema em abordagem, os quais poderão ter implicações graves numa utilização operacional, para fins de gestão.

Estamos conscientes que os assuntos abordados são um tema de discussão sensível, envolvendo conceitos e abordagens que, apesar de serem correntes em projectos internacionais em desenvolvimento na margem norte do Mediterrâneo (ex.: ASTIMWR, LISSE, DEMETER, etc.), não têm o enquadramento adequado em relação à importância do tema. 


\section{AGRADECIMENTOS}

A todos os colegas do IHERA/IDRHa, que permitiram dar corpo a muito do conteúdo do tema. Aos Eng. Pedro Oliveira, Drs Jorge Rocha e Paulo Morgado, por terem possibilitado a implementação de desenvolvimentos sobre o tema. À ABCaia, na pessoa do Eng. Aristides Chinita, por nos ter permitido aplicar e desenvolver o tema de forma operativa. Ao Dr. Jean Paul Gilg e Dr. ${ }^{a}$ Helène Geryonamis (EHSS-Paris), pelo grande apoio no desenvolvimento e sedimentação dos conceitos e temas apresentados.

\section{BIBLIOGRAFIA}

Allen, R. G.; Pereira, L. S.; Raes, D. e Smith, M. (1998) - Crop evapotranspiration, guidelines for computing crop water requirements. FAO Irrigation and Drainage Paper 56.

Bastiannssen, V.; Vanderwal, T. e Visser, T. (1996) - Diagnosis of regional evaporation by remote sensing to support irrigation performance assessment. Irrigation and drainage system, 10: 1-23.

D'URSo, G. (2001) - Simulation and management of on-demand irrigation systems: a combined agrohydrological and remote sensing approach. Wageningen University, Wageningen. 193 p.

Menenti, M. (2000) - Evaporation. Remote sensing and water management. Springer: 157-188.

Oliveira, P. A. (2000) - Desenvolvimento de um Sistema de Informação Geográfica para a gestão de um perímetro de rega. ISA, Lisboa.

Perdigão, A. et al (2001) - Desenvolvimento de um sistema de apoio à gestão da informação geográfica no perímetro de rega do Caia. IHERA, Lisboa. 